\title{
New algorithm for a robust user-independent evaluation of beginning instability for the experimental FLC determination
}

\section{Journal Article}

Author(s):

Hora, Pavel; Volk, Wolfram

Publication date:

2011-09

Permanent link:

https://doi.org/10.3929/ethz-b-000068442

Rights / license:

In Copyright - Non-Commercial Use Permitted

Originally published in:

International Journal of Material Forming 4(3), https://doi.org/10.1007/s12289-010-1012-9 


\title{
New algorithm for a robust user-independent evaluation of beginning instability for the experimental FLC determination
}

\author{
W. Volk • Pavel Hora
}

Received: 27 April 2010 / Accepted: 26 November 2010 / Published online: 24 December 2010

(C) Springer-Verlag France 2010

\begin{abstract}
The failure prediction in sheet metal forming is typically realized by evaluating the so called forming limit curves (FLC). The standard experimental method is the Nakajima test, where sometimes also the Marciniak test setup is used. Up to now, the FLC determination was performed with failed specimens and an one-directional intersection line method or by manually analyzing the estimated strains before cracking. Both methods determine the failure by considering the occurrence of cracking and do not consider the possibility of time continuous recording of the Nakajima test. Consequently forming limit curves which have been evaluated in such way are often "laboratory dependent" and deviate for identical materials significantly. This paper presents an algorithm for a fully automatic and time-dependent determination of the beginning plastic instability based on physical effects. The algorithm is based on the evaluation of the strain distribution based on the displacement field which is evaluated by optical measurement and treated as a mesh of a finite element calculation. The critical deformation
\end{abstract}

\section{W. Volk}

BMW Group, Forming Technology,

Product and Process Planning,

Munich, Germany

e-mail: wolfram.volk@bmw.de

URL: www.bmwgroup.com

\section{P. Hora $(\bowtie)$}

Institute of Virtual Manufacturing, ETH Zurich,

Tannenstrasse 3,

8092 Zurich, Switzerland

e-mail: hora@ivp.mavt.ethz.ch

URL: www.ivp.ethz.ch states are then defined by 2D-consideration of the strain distribution and their time derivates using a numerical evaluation procedure for detecting the beginning of the localization. The effectiveness of the proposed algorithm will be presented for different materials used for the Numisheet'08 Benchmark-1 with Nakajima test.

Keywords FLC · Local necking - Optical detection of strain localizations $\cdot$ Nakajima-test

\section{Introduction}

The forming limit curve (FLC) was established as one of the most utilized tools in detecting failure due to necking or cracks in FE-simulation. The curves of experimental methods are normally obtained with Nakajima or Marciniak [1] tests and have to be accomplished under laboratory-like conditions. The ISO standardization is given in [1]. Both of these methods are based on experimental series where gridded samples are being deformed and simultaneously the position of the intersection of the grid lines is being recorded with photogrammetrical methods (Fig. 1). Typical boundary conditions are punch velocity of $1 \mathrm{~mm} / \mathrm{s}$ and recording frequency of 10 pictures per second.

By using different sample geometries, various strain conditions can be traced to get the corresponding points in the FLD (Fig. 2). $\beta$ denotes the strain ratio

$\beta=\frac{\varphi_{2}}{\varphi_{1}}$

Currently, different methods are used in industrial practice for the identification of the FLC's (Fig. 3). 
Fig. 1 Nakajima test setup. Optical measurement system [2]
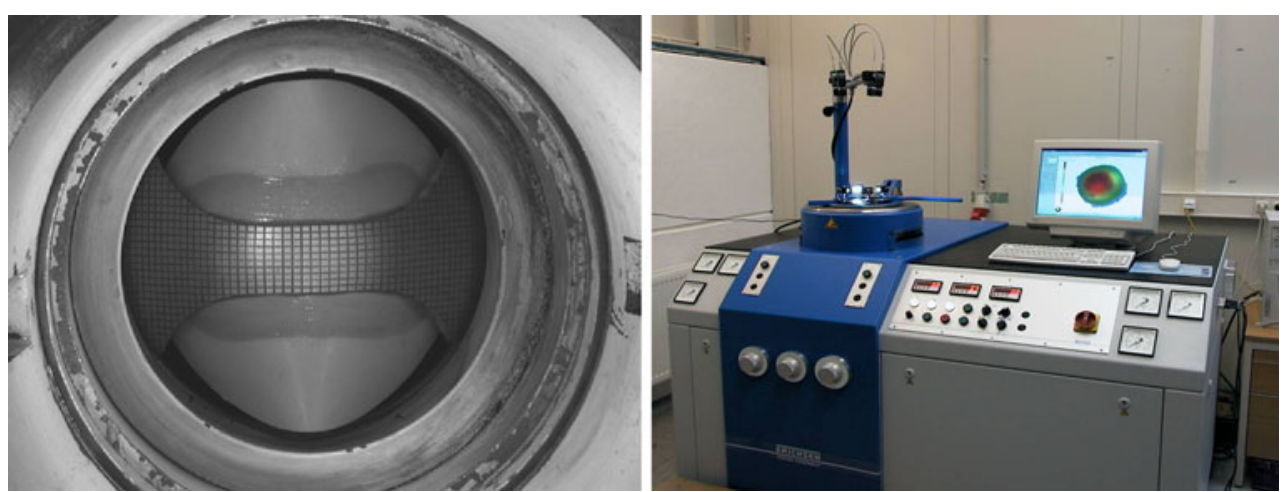

The 0d-method considers usually the last detected deformation state ("picture") before the crack occurs and defines the achieved maximal principal strain as the critical strain value.

The ISO definition identifies strain distribution along a cross section (1d-method). The FLD major strain value is defined at the position where the second derivate of $\varphi_{m a j}$ reaches the maximum. This definition is mathematically clear but has no physical background.

Therefore, in the present paper, an alternative will be presented which calculates the occurrence of plastic instability on the basis of the recorded time-dependent crosspoint position data from the experiments and evaluate the strain distribution in a $2 \mathrm{D}$ section (2d-method).

\section{Evaluation of the strain and strain rate distributions}

Input data and finite element approach

The only required input data for the new method is a full set of global coordinates of the grid line crosspoints, which can be handled like global coordinates of a finite element mesh, see Fig. 4.

Following this idea all interesting physical quantities (strains, strain rate etc.) can be calculated by using well established methods of FE theory. If the test data is obtained with a regular grid pattern the crosspoints of the grid can be directly used as the vertices of the FE mesh. If crosspoints of the optical measurement are missing the coordinates of the voids can be calculated by suitable mathematical methods, e.g. [3] or [4]. If stochastic spray structures are applied for the optical measurement an additional preparation step to generate the grid pattern before starting the evaluation process is necessary.

\section{Calculation of strains}

For the determination of the forming limit strains a Total Lagrange description is used, where the initial mesh is taken as reference configuration $X_{k}$ and the current picture as present configuration $x_{k}$. The strains are evaluated using classical FEM 4-node membrane element theory, described for example by [5].

Following this the linear Cauchyian strain tensor can be described with:

$\varepsilon_{i j}=\frac{1}{2}\left(H_{i j}+\left(H_{i j}\right)^{T}\right)$

where

$H_{i j}=\frac{\partial u_{i}}{\partial x_{j}}=J^{-1} \frac{\partial N_{i}}{\partial \xi_{\alpha}}\left(x_{j}-X_{j}\right)=J^{-1} \frac{\partial N_{i}}{\partial \xi_{\alpha}} u_{j}$
Fig. 2 Nakajima specimen shapes (W. Hotz, FLC-Zurich). Dependency of sample geometry from strain path
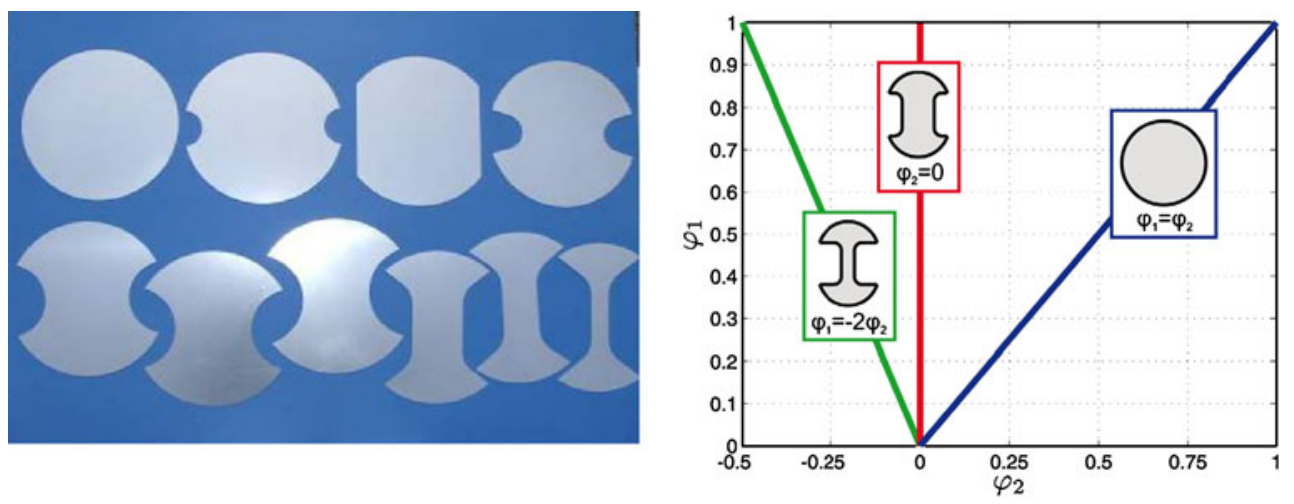


\section{D-Method [2]}

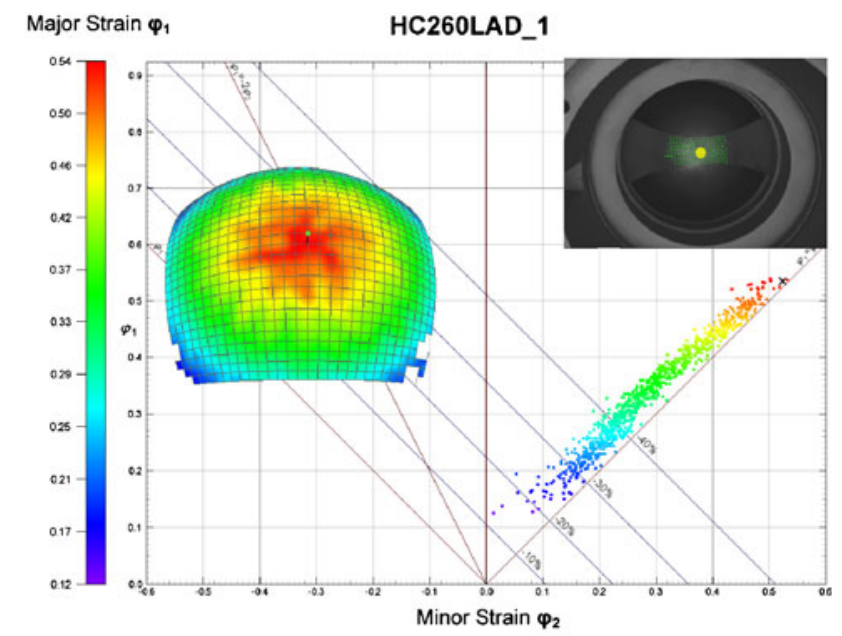

1D-Method [1]

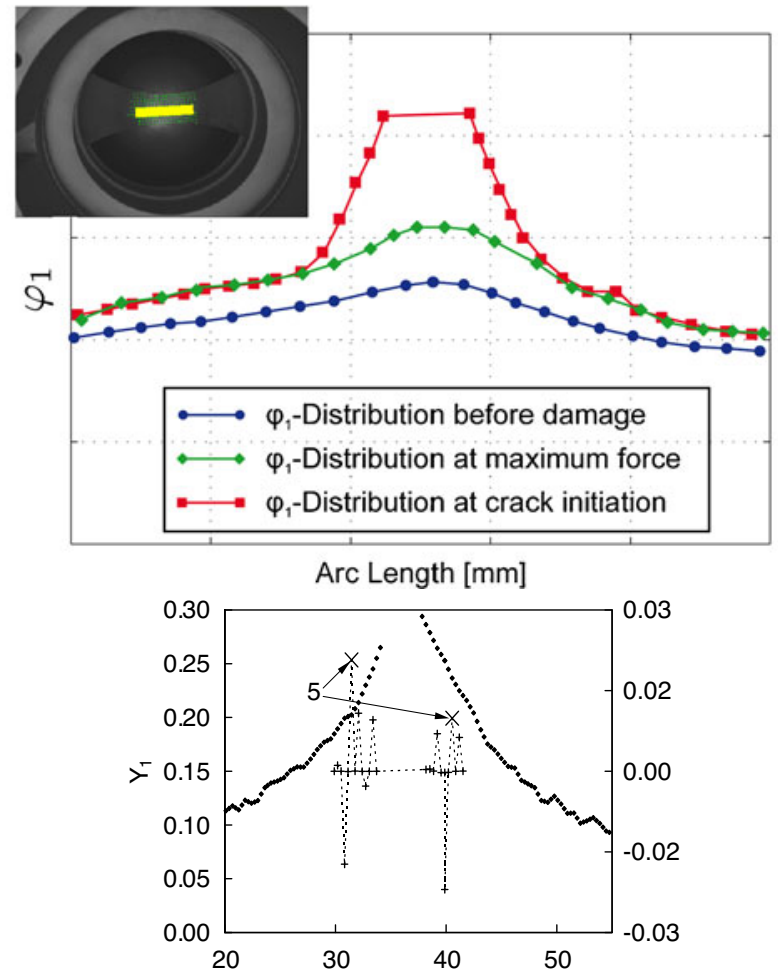

Fig. 3 Different areas of the FLC evaluation methods

Therein $u_{i}$ is the displacement vector, $J^{-1}$ is the inverse of the Jacobian and $N_{i}$ the isoparametric functions $N_{i}=1 / 4\left(1 \pm \xi_{1}\right)\left(1 \pm \xi_{2}\right)$.

The plane principal strains result from the characteristic equation $\operatorname{det}\left(\varepsilon_{i j}-\lambda \delta_{i j}\right)=0$ with:

$$
\begin{aligned}
& \varepsilon_{1}=\frac{1}{2}\left(\varepsilon_{11}+\varepsilon_{22}+\sqrt{\left(\varepsilon_{11}-\varepsilon_{22}\right)^{2}+4 \varepsilon_{12}{ }^{2}}\right. \\
& \varepsilon_{2}=\frac{1}{2}\left(\varepsilon_{11}+\varepsilon_{22}-\sqrt{\left(\varepsilon_{11}-\varepsilon_{22}\right)^{2}+4 \varepsilon_{12}{ }^{2}}\right.
\end{aligned}
$$

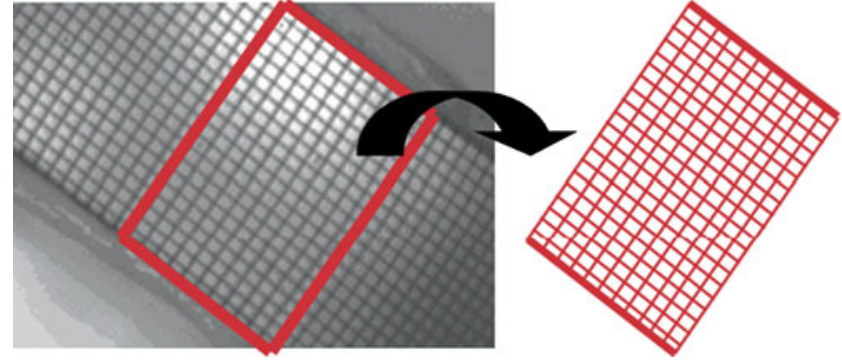

Fig. 4 Interpretation of measurement grid as finite element mesh

Ultimately the Henckyian strain gauge is applied due to large strain components because of the Total Lagrange description:

$$
\begin{aligned}
& \varphi_{1}=\ln \left(1+\varepsilon_{1}\right) \\
& \varphi_{2}=\ln \left(1+\varepsilon_{2}\right) \\
& \varphi_{3}=-\left(\varphi_{1}+\varphi_{2}\right)
\end{aligned}
$$

With these strain components, the points in the FLC are determined.

\section{Calculation of strain rates}

For the determination of the strain rates and especially of the thinning rate, it is necessary to take the numerical derivative of the deformation gradient with respect to time into account. The determination of the deformation gradient tensor on the basis of the displacement gradient is performed as follows:

$F_{i j}=\left(I+H_{i j}\right)$

Subsequently, with the derivative of the deformation gradient, the velocity gradient tensor is figured out according to the following formula [6]:

$L_{i j}=\frac{\partial F_{i j}}{\partial t} F_{i j}^{-1}=\dot{F}_{i j} F_{i j}^{-1}$

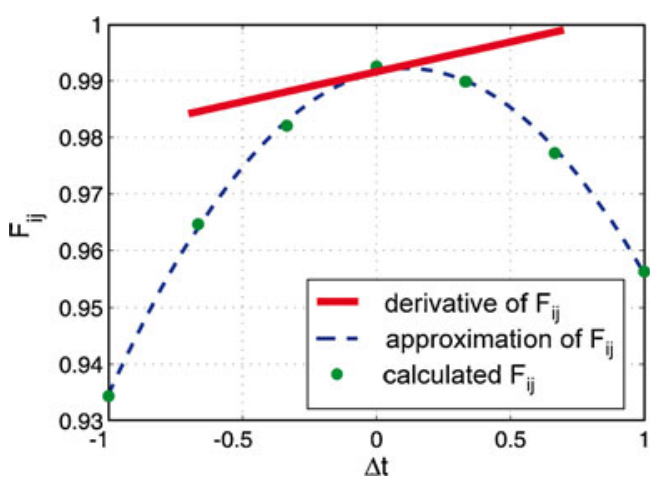

Fig. 5 Determination of $\dot{F}_{i j}(t)$ by using quadratic least square interpolation with seven time history points 
Fig. 6 Development of the localized necking by the reduction of the plastic zone to the shear band(s)
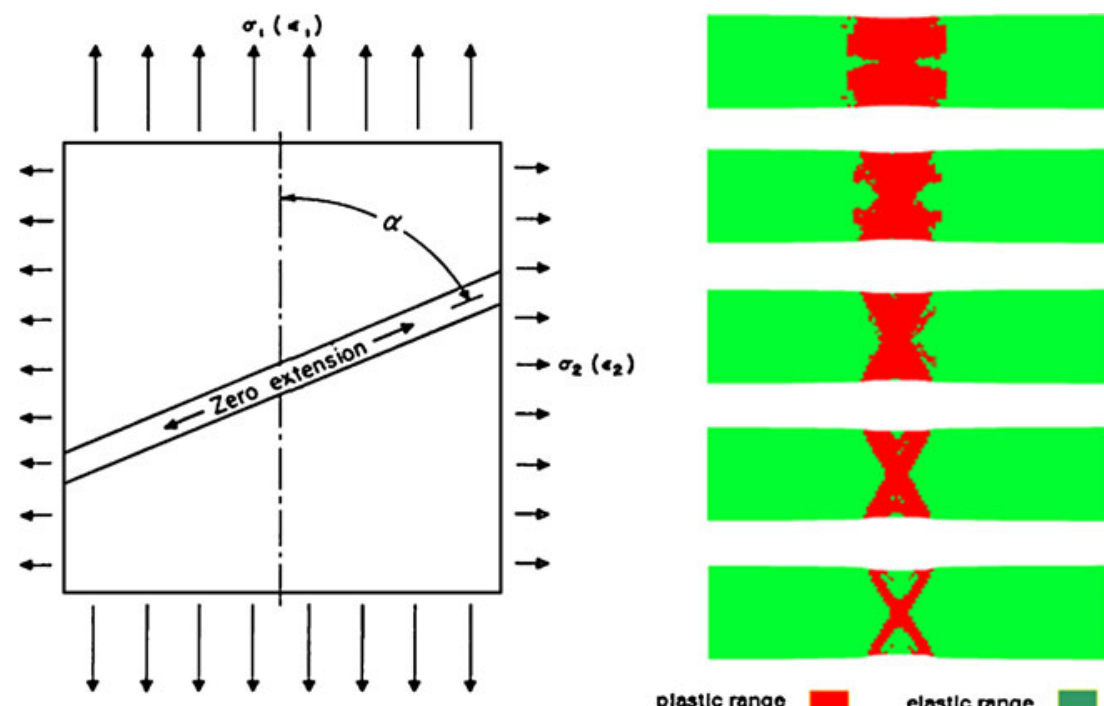

plastle range and hence, the deformation rate tensor can be computed:

$D_{i j}=\frac{1}{2}\left(L_{i j}+L_{i j}{ }^{T}\right)$

Finally, the plane principal components of the deformation rate are evaluated by computing the eigenvalues $D_{l}$ and $D_{2}$ of the deformation rate tensor $D_{i j}$. To calculate the necessary thinning rate, the deviatoric character of $D$ is used:

$\dot{\varepsilon}:=\left|D_{3}\right|=-\left(D_{1}+D_{2}\right)$

The thinning rate $\dot{\varepsilon}$ is the main physical quantity to identify the picture with beginning instability and calculating the strain values for the entries of the FLC.

The numerical derivative of the deformation gradient will be very un-accurate, if no smoothing over the time steps is applied. For those reasons in [3] a quadratic interpolation over time with typically seven time history points is proposed for the deformation gradient $F_{i j}(t)$, see Fig. 5. Alternatively one can use Bézier-splines or Bsplines interpolation methods [7].

\section{Algorithm for detection of the forming limit states}

Plastic instability

From a continuum mechanical point of view, the plastic instability is the local concentration of the remaining plastic deformation in small (shear-) bands and a fall-back of the other areas in the elastic range (Fig. 6).
Hence, two effects can be noticed, namely the strain inside this band increases on the one hand and on the other hand it has to remain roughly constant outside. This is displayed in Fig. 7. It is remarkable in that figure that the characteristics of thinning over time seem to grow relatively continuously, which can cause problems for an automatic detection of beginning plastic instability.

Therefore, it is helpful to have a look at the distribution of the thinning rate as shown in Fig. 8. It demonstrates that the described effect is much more distinct if the strain rate, or rather the thinning rate, is in focus. This is due to the fact that the thinning rate outside of the instability area has to decrease, which intensifies even more the effect of an increase inside this area. In the given example one can now identify the beginning instability (marked with white arrow).
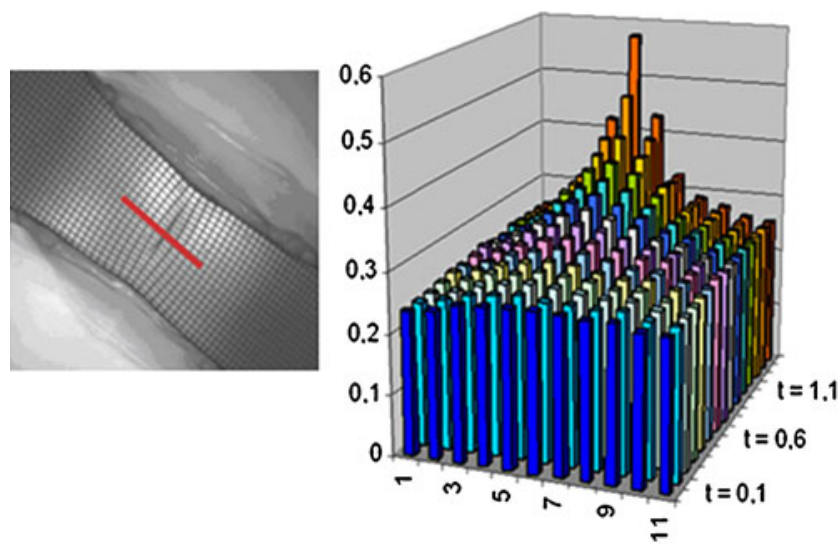

Fig. 7 Time dependent evolution of the necking. Mutation of negative thinning over time until the beginning of instability [8] 

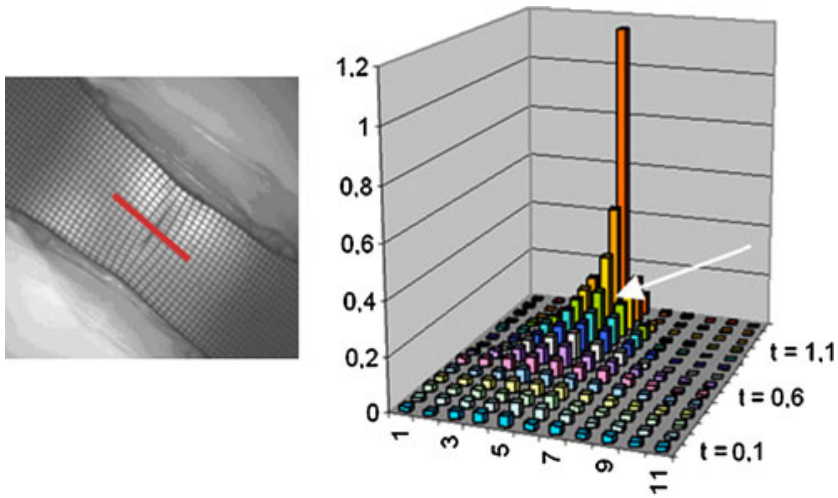

Fig. 8 Time dependent evolution of the necking. Mutation of negative thinning rate over time until the beginning of instability [8]

Consequently, the following detailed approach is based on finding a robust criterion to detect the beginning of instability by accounting for the distribution of the thinning rate.

Detection of beginning plastic instability

For the detection of beginning instability it can be used the two main characteristic effects of localized necking. These are the local concentration of remaining plastic deformation and the acceleration of thinning rates in the instability zone until the crack occurs. In [3] the so-called frequency diagram was introduced and in [7] an integral value method is proposed with taking into account the developing geometrical differences between stable and instable areas. The following algorithm combines the advantages of these two methods, the clear physical motivation combined with an easy implementation.

\section{Identification of necking area}

The first step of the algorithm is the identification of a sufficient number of elements which are sure in the instability zone. Therefore $\Gamma^{k}$ is introduced as set of sorted thinning rates $\dot{\varepsilon}_{j}^{k}$ for all elements $j$ of the analysis area and every picture $k$.

$$
\begin{gathered}
\Gamma^{k}:=\left\{\dot{\varepsilon}_{1}^{k}, \dot{\varepsilon}_{2}^{k}, \ldots ., \dot{\varepsilon}_{n}^{k}\right\} \text { with } \dot{\varepsilon}_{i-1}^{k} \leq \dot{\varepsilon}_{i}^{k} \text { for } i=2, \ldots ., g \\
\text { and } k=1, \ldots ., b
\end{gathered}
$$

Therein $g$ is the number of elements of the analysis area and $b$ the number of pictures. It is recommended to take about 30-40 pictures to ensure a sufficient database for the identification of the beginning instability. The representative maximum thinning rate $\dot{\bar{\varepsilon}}_{\text {max }}$ is now defined as the arithmetic mean value of the five highest thinning rates in the second last picture

$\dot{\bar{\varepsilon}}_{\max }=1 / 5 \sum_{i=g-4}^{i=g} \dot{\varepsilon}_{i}^{b-1}$

It is recommended not to take the last picture before the crack occurs due to slight numerical problems in the determination of the time derivative, see Fig. 5. Every element with a thinning rate higher than $\alpha \dot{\bar{\varepsilon}}_{\max }$ in the second last picture is identified as element in the necking zone. The set $\mathrm{N}$ is the union of all of these elements and contains of $n$ elements. The elements of the sets $N^{k}$ are the thinning rates of all elements of $\mathrm{N}$ for every picture $k$.

$$
\begin{aligned}
& \mathrm{N}=\forall \text { Elem with } \dot{\varepsilon}_{i}^{b-1} \geq \alpha \dot{\bar{\varepsilon}}_{\text {max }} \\
& \mathrm{N}^{k}=\left\{{ }^{\mathrm{N}} \dot{\varepsilon}_{1}^{k},{ }^{\mathrm{N}} \dot{\varepsilon}_{2}^{k}, \ldots,{ }^{\mathrm{N}} \dot{\varepsilon}_{n}^{k}\right\}
\end{aligned}
$$

The factor $\alpha$ should be chosen that the set $N^{k}$ contains of 7 to 15 elements for a $1 \mathrm{~mm}$ grid and 5 to 10 elements for a $2 \mathrm{~mm}$ grid. Experience has shown that this can be obtained with $\alpha$ between 0.5 and 0.6. In Fig. 9 three examples are shown of the identified necking area for three different strain paths (uni-axial, plain strain, bi-axial).

\section{Identification of beginning instability}

The next step in the algorithm is the identification of beginning instability. Therefore the representative thinning
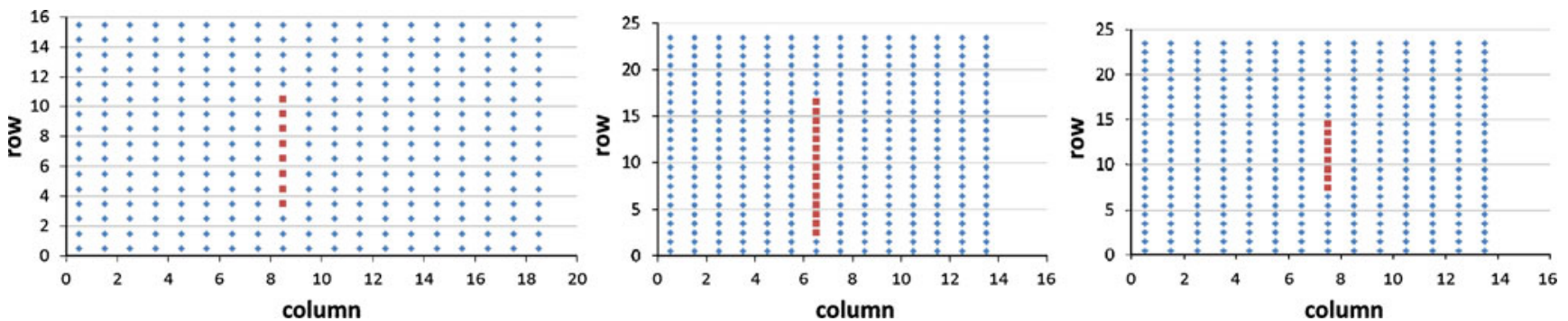

Fig. 9 Identified necking areas $\mathrm{N}$ of $\mathrm{HC} 220 \mathrm{YD}, 1.6 \mathrm{~mm}$ (Numisheet 2008 Benchmark material), for uni-axial $(\beta \approx-0.5$, left $)$, plain strain $(\beta \approx 0$, middle) and bi-axial strains ( $\beta \approx 1$, right), $\alpha=0.6$ 


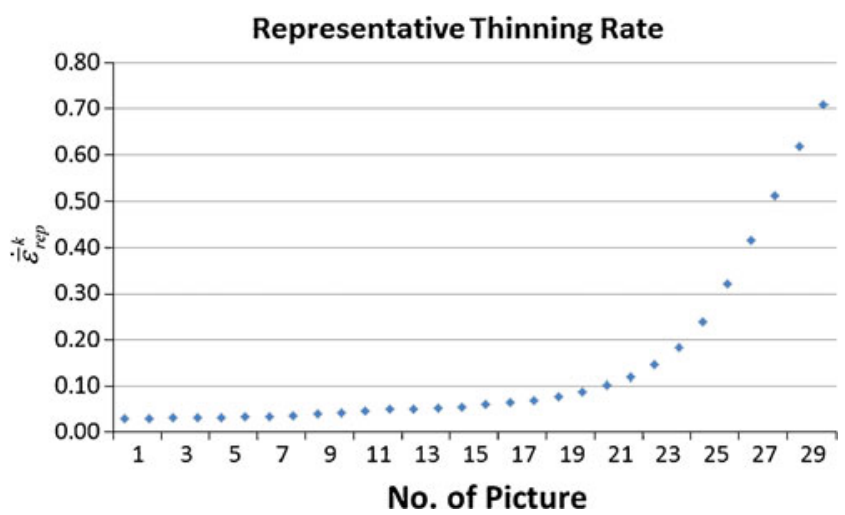

Fig. 10 Representative thinning rate $\dot{\bar{\varepsilon}}_{\text {rep }}^{k}$ of last 30 pictures before crack, plain strain specimen, HC220YD, $1.6 \mathrm{~mm}$, Numisheet 2008 Benchmark material

rate $\dot{\bar{\varepsilon}}_{r e p}^{k}$ is introduced as arithmetic mean value of all elements of every set $N^{k}$ for every picture $k$.

$\dot{\bar{\varepsilon}}_{\text {rep }}^{k}=1 / n \sum_{i=1}^{i=n} \mathrm{~N}^{k} \dot{\varepsilon}_{i}^{k}$

In Fig. 10 the set of $\dot{\bar{\varepsilon}}_{\text {rep }}^{k}$ (last 30 pictures before the crack occurs) is plotted for plain strain condition of the HC220YD Numisheet Benchmark material. The representation of the diagram is characteristic: A nearly linear increase with low slope at the beginning, a linear increase with high slope at the end and a curved area in between.

The interpretation of this characteristic behaviour is obvious. At the beginning one can see the stable nearly homogeneous deformation and the localized necking as instable deformation at the end until the crack occurs. In Fig. 11 it is shown that this typical behaviour is also remarkable for uniaxial strain $(\beta \approx-0.5)$ and biaxial strain $(\beta \approx 1.0)$.

The difference between the strain conditions is the characteristic occurrence of the curved area, which is also a measurement for the different amount of diffuse necking. Due to the fact that all strain conditions show the presented typical behaviour it is now used for the detection of beginning instability. The stable and instable areas are fitted with two linear curves by using the least square method. The picture which is next to the intersection of these two straight lines will be defined as beginning instability, see Fig. 12.

For the identification of the stable area it is recommended to start with the fourth picture because of the determination of the time derivative with seven history points, see Fig. 5. Then the sequence of linear curve fitting is applied with at least four points up to the maximum number of available points. The coefficients of the fitted linear curve can be calculated easily with the Gaussian normal form.

$$
\begin{aligned}
& \dot{\bar{\varepsilon}}_{s t}^{r}(k)=a_{s t}^{r}+b_{s t}^{r} \cdot k \\
& \left(\begin{array}{c}
a_{s t}^{r} \\
b_{s t}^{r}
\end{array}\right)=\left(\mathbf{A}_{s t}^{r T} \mathbf{A}_{s t}^{r}\right)^{-1} \mathbf{A}_{s t}^{r T} \mathbf{c}_{s t}^{r}, r=4, \ldots, b-3 \\
& \left(A_{s t}^{r}\right)_{j 1}=1, j=1, \ldots, r ; \quad\left(A_{s t}^{r}\right)_{j 2}=j+3, j=1, \ldots ., r \\
& \left(c_{s t}^{r}\right)_{j}=\dot{\bar{\varepsilon}}_{r e p}^{j+3}, j=1, \ldots, r
\end{aligned}
$$

Therein $b$ is the number of available pictures, $a_{s t}^{r}$ and $b_{s t}^{r}$ are the coefficients of the linear curve fitting for the stable area with taking $r$-pictures into account starting from the fourth one. With the coefficients $a_{s t}^{r}$ and $b_{s t}^{r}$ one can define the sequence of mean least square differences $d_{s t}^{r}$.

$d_{s t}^{r}=1 /(r+2) \sum_{i=-2}^{i=r}\left(a_{s t}^{r}+(i+3) \cdot b_{s t}^{r}-\dot{\bar{\varepsilon}}_{\text {rep }}^{i+3}\right)^{2}$

The minimum of the sequence $d_{s t}^{r}$ denotes the coefficients $a_{s t}^{r_{\min }}$ and $b_{s t}^{r_{\min }}$ for the linear curve fitting of the stable area of $\dot{\bar{\varepsilon}}_{\text {rep }}^{k}$.

$d_{s t}^{r_{\min }}=\operatorname{Min}\left(d_{s t}^{r}\right), r=4, \ldots, b-3$

In the same manner the linear curve fitting of the instable area of $\dot{\bar{\varepsilon}}_{\text {rep }}^{k}$ can be realized getting the coefficients $a_{i n}^{s_{\min }}$ and $b_{i n}^{s_{\min }}$. The only difference is to take the last picture as starting point of the approximation, due to the fact that only
Fig. 11 Representative thinning rate $\dot{\bar{\varepsilon}}_{\text {rep }}^{k}$ for uniaxial strain (left) and biaxial strain (right) specimen, HC220YD, $1.6 \mathrm{~mm}$, Numisheet 2008 Benchmark material
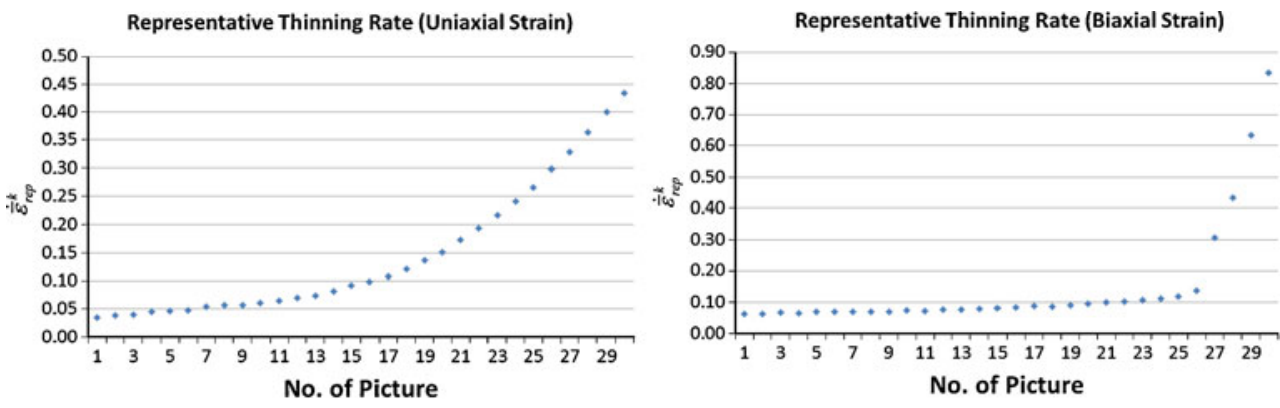


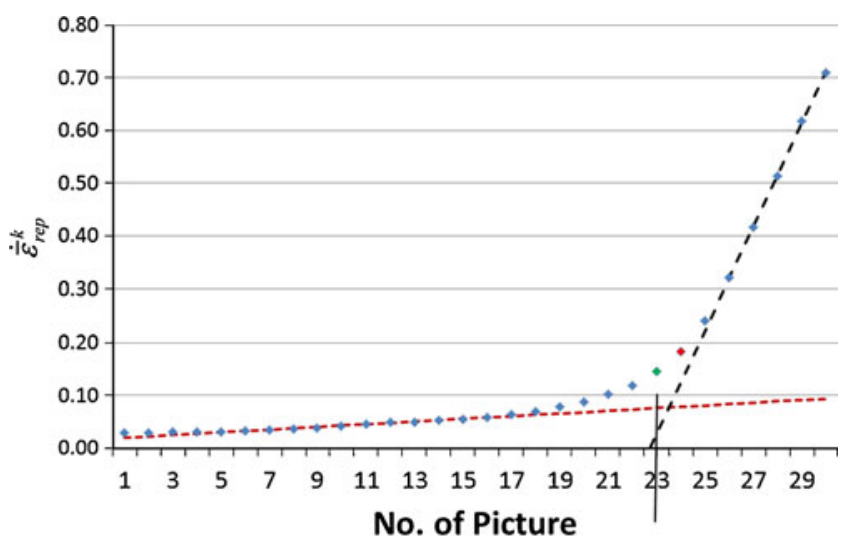

Fig. 12 Detection of beginning instability with linear curve fitting using least square method, first instable picture for the example is identified as number 24, last stable one is number 23

a limited number of pictures in the instable area is available and the numerical deviations of the time derivative can be accepted.

$\dot{\bar{\varepsilon}}_{i n}^{s}(k)=a_{i n}^{s}+b_{i n}^{s} \cdot k$

$\left(\begin{array}{c}a_{i n}^{s} \\ b_{\text {in }}^{s}\end{array}\right)=\left(\mathbf{A}_{\text {in }}^{s}{ }^{T} \mathbf{A}_{\text {in }}^{s}\right)^{-1} \mathbf{A}_{\text {in }}^{s}{ }^{T} \mathbf{c}_{\text {in }}^{s}, s=3, \ldots, b$

$\left(A_{i n}^{s}\right)_{j 1}=1, j=1, \ldots, s ; \quad\left(A_{i n}^{s}\right)_{j 2}=b-s+j, j=1, \ldots \ldots, s$

$\left(c_{i n}^{s}\right)_{j}=\dot{\bar{\varepsilon}}_{\text {rep }}^{b-s+j}, j=1, \ldots, s$

$d_{i n}^{s}=1 / s \sum_{i=1}^{i=s}\left(a_{i n}^{s}+(b-s+i) \cdot b_{i n}^{s}-\dot{\bar{\varepsilon}}_{\text {rep }}^{b-s+i}\right)^{2}$

$d_{i n}^{S_{\min }}=\operatorname{Min}\left(d_{i n}^{s}\right), s=3, \ldots, b$

The crosspoint $k_{\text {crit }}$ of the linear curve fittings for the stable and instable area is given by

$k_{c r i t}=\frac{a_{i n}^{s_{\min }}-a_{s t}^{r_{\min }}}{b_{s t}^{r_{\min }}-b_{\text {in }}^{s_{\min }}}$.
The first picture with beginning instability $k_{\text {inst }}$ is defined as the picture which is next to $k_{c r i t}$ and the previous picture as the last stable one $k_{\text {stab }}$.

$k_{\text {inst }}=\operatorname{Int}\left(k_{\text {crit }}+0.5\right) ; k_{\text {stab }}=k_{\text {inst }}-1$

\section{Calculation of strain values for the FLC}

The calculation of strain values for the entries in the FLC is the last step in the presented algorithm. The mean values of the principle strains $\varphi_{1}^{k_{s t a b}}$ and $\varphi_{2}^{k_{\text {stab }}}$ of all elements of the set $\mathrm{N}$ (see Formula 12) give a unique definition for the entries of the FLC for the identified picture $k_{\text {stab }}$.

$$
\begin{aligned}
\varphi_{1}^{F L C} & =1 / n \sum_{i=1}^{i=n} \mathrm{~N} \varphi_{1}^{k_{\text {stab }}} \\
\varphi_{2}^{F L C} & =1 / n \sum_{i=1}^{i=n}{ }^{N} \varphi_{2}^{k_{\text {stab }}}
\end{aligned}
$$

\section{FLC-evaluation examples}

Figure 13 demonstrates for the Numisheet BM1 materials HC220YD with thicknesses of $0.8 \mathrm{~mm}$ and $1.6 \mathrm{~mm}$ as well as the HC260LAD (1.1 mm thickness) the strengths of the proposed $2 \mathrm{D}$ evaluation method. Because neither the position on the specimen nor the time point has to be fixed manually, the procedure is very robust. The Nakajima experiments executed by two different labs deliver very similar FLD results. For the $1.6 \mathrm{~mm}$ material laboratory 1 has used a mesh with $2 \mathrm{~mm}$ grid size and laboratory 2 a mesh with $1 \mathrm{~mm}$ grid size. Therefore the very slight differences between the two FLD curves can be explained by the different resolutions. Nevertheless the robustness of the algorithm is remarkable even for different grid sizes (see Fig. 13).
HC220YD, $0.8 \mathrm{~mm}$

$\varphi_{1}$

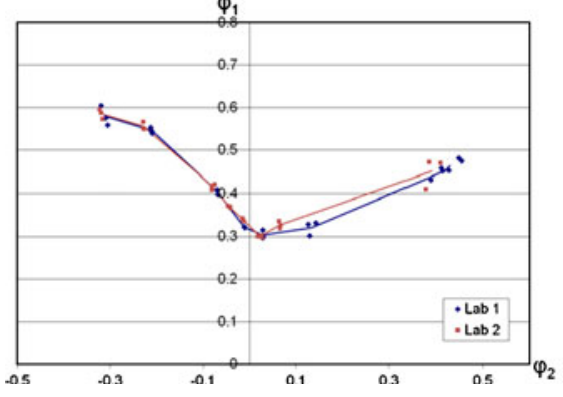

HC220YD, $1.6 \mathrm{~mm}$

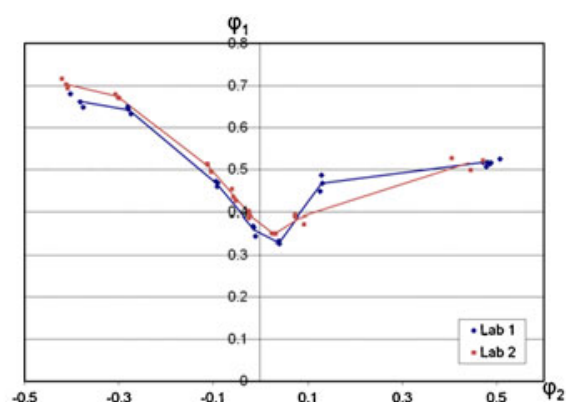

HC260LAD, $1.1 \mathrm{~mm}$

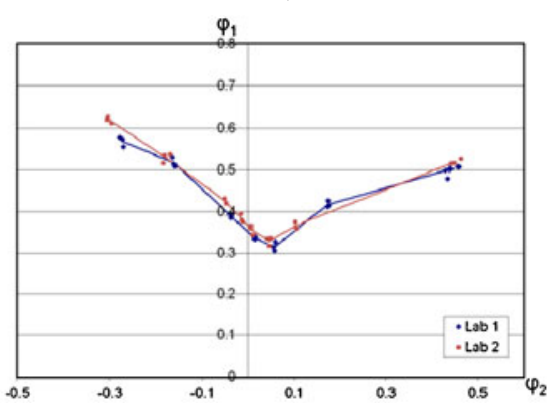

Fig. 13 Detection of the FLC. Comparison of different materials, laboratories and mesh sizes 


\section{Conclusions and outlook}

The new evaluation method presented here has two crucial benefits. On the one hand, it allows a fully automatic derivation of the FLC on the basis of experimental testing results and, on the other hand, it is now possible to use the time continuous recording of the testing machines to obtain the beginning of plastic instability and therefore the first occurrence of material failure. The algorithm is clear, easy to implement and bases on a physical motivation. The robustness has been proven by numerous tests in different laboratories even with different mesh sizes. It seems to be interesting to compare the obtained experimental results with theoretical and numerical investigations, e.g. by evaluating the so called acoustic tensor [9].

Acknowledgement The authors would like to thank B. Eberle for his excellent work during his master thesis [7] which was the basis for the presented evaluation method.

\section{References}

1. Metallic materials -Sheet and strip-Determination of forming limit curves - Part 2: Determination of forming limit curves in laboratory (ISO/DIS 12004-2). ISO copyright office, 2006
2. Formänderungsanalyse-System AutoGrid ${ }^{\circledR}$ : Benutzerhandbuch. ViALUX Messtechnik + Bildverarbeitung GmbH

3. Volk W (2006) New experimental and numerical approach in the evaluation of the FLD with the FE-method. FLC-Zurich'06 conference on Numerical and experimental methods in prediction of forming limits in sheet forming and tube hydroforming processes, pp 26-30

4. Eberle B, Volk W, Hora P. Automatic approach in the evaluation of the experimental FLC with a full $2 \mathrm{~d}$ approach based on a time depending method. NUMISHEET'08 - Proceedings of the 7th Int. Conf. and Workshop on Numerical Simulation of 3D Sheet Metal Forming Processes, Part A, pp 279-284, Switzerland, ISBN 978-3909386-80-2

5. Bathe K-J (1995) Finite Element Procedures. Springer Verlag, ISBN 0133014584

6. Altenbach J, Altenbach H (2006) Einführung in die Kontinuumsmechanik. B. G. Teubner Stuttgart

7. Eberle B (2008) Entwicklung eines robusten numerischen Verfahrens zur automatischen Bestimmung experimenteller FLC-Kurve. Master thesis, Inst. of Virtual Manufacturing, ETH-Zurich

8. Volk W. Benchmark 1 - Virtual Forming Limit Curve. NUMISHEET'08 - Benchmark Proceedings of the 7th Int. Conf. and Workshop on Numerical Simulation of 3D Sheet Metal Forming Processes, Part B, pp 3-42, Switzerland,. ISBN 978-3-909386$80-2$

9. Bigoni D, Zaccaria D (1994) On the eigenvalues of the acoustic tensor in elastoplasticity. Eur J Mech A Solids 13(5):621638 